\title{
A probabilistic model for the discrimination of visual number
}

\author{
MICHIEL P. van OEFFELEN and PETER G. VOS \\ University of Nijmegen, Nijmegen, The Netherlands
}

\begin{abstract}
This paper proposes a probabilistic model of how humans identify the number of dots within a briefly presented visual display. The model is an application of Thurstone's law of comparative judgment, and it is assumed that the internal representation of numerosity consists of log. spaced random variables. The discrimination between any two different numerosities is consequently described as a function of $\max / \mathrm{min}$, where max and min are the larger and smaller numbers, respectively. The model was tested in two experiments in which the Weber fraction for numerosity, corresponding with the critical ratio of $\max$ and $\min$, was found to have the value of .162. It was concluded that the classical span of subitizing numerosity is but a special case of the span of discrimination.
\end{abstract}

If one is asked to estimate under time pressure the number (n) of dots in a display, response accuracy appears to be high when the number of dots does not exceed about 6 , but decreases rapidly for larger values of $\mathrm{n}$. Under self-paced task conditions, response latencies show a similar pattern. Latencies are very fast for small numbers and slow down considerably for $\mathrm{n}$ larger than about 6. Taves (1941) studied judgments of numerousness and stated that two mechanisms were involved-one used for up to 7 dots, the other for larger fields. For the first mentioned discriminatory process, Kaufman, Lord, Reese, and Volkman (1949) proposed the term "subitizing."

Bourdon (1908) suggested that small numbers of dots are apprehended by immediate cognition: " 1,2 , 3,4 are thus sensations just like green, red, round, or square; the quality of twoness of a group of objects is essentially perceived in the same way as the quality red or round" (p. 430; translation by the authors). There are certainly reasons for doubting the supposition that subitizing is some sort of purely holistic information processing as Bourdon suggested. One such reason is the frequently reported finding that latencies also tend to increase within the subitizing range. Woodworth and Schlosberg (1954) noticed that the differences in question fit well with what is known about choice reaction times: "The bigger a difference, the more quickly it is perceived; and the (relative) difference between 1 and 2 is greater than that between 2 and 3 , and so on up the scale. In

Portions of this paper benefited from discussions with Prof. Dr. E. Eijkman. The authors wish to thank an anonymous reviewer. This research was supported by a grant from The Netherlands Organization for the Advancement of Pure Research (Zuiver Wetenschappelijk Onderzoek). The authors' mailing address is: Psychology Department, KUN, Montessorilaan 3, $6500 \mathrm{HE}$ Nijmegen, The Netherlands. identifying 5 dots you have to distinguish this number from 4 and 6; identifying 2 dots, you need only make the easier discrimination between 2 dots and 1 and 3" (p. 98). This hypothesis was considered further by Averbach (1963) in a study on the span of visual apprehension. He suggested that the discrimination of visual number may depend on the ratio of the difference in magnitude between two numbers and the magnitude of an actually presented number. If that ratio is at least as large as the (hypothetical) Weber fraction for visual number, then, according to Averbach, judgmental accuracy and speed would have the characteristics attributed to subitizing number.

Moyer and Landauer (1967) measured reaction time for deciding which of two simultaneously presented digits was larger and observed that reaction time decreased monotonically as the difference between the two numbers increased. In interpreting their data, they suggested that the displayed numerals were converted to analogue magnitudes, and a comparison was then made between the magnitudes in much the same way that comparisons are made between physical stimuli. The "internal magnitude" should then be a nonlinear compressed function of the magnitude of the digit. In such compressive spacing, 8 and 9 would be closer together on the internal scale than 7 and 8,7 and 8 closer together than 6 and 7 , and so on.

The present paper proposes a simple theory for the discrimination of visual number. The theory essentially is an application of Thurstone's law of comparative judgment (Thurstone, 1927; Torgerson, 1958).

\section{Model of Number Discrimination}

We consider stimuli consisting of sets of $\mathrm{n}$ dots. All physical aspects of the dots, such as brightness and area, are kept constant throughout. Moreover, ar- 
rangement of dots within a stimulus is considered to be random. Hence, a stimulus is described by the number, $n$, of dots only.

We suppose that the discriminability of such stimuli obeys Thurstone's law of comparative judgment (Thurstone, 1927; Torgerson, 1958). Briefly summarized, this law states that a (presented) stimulus triggers a discriminal process in which the external stimulus value $n$ is transformed into some value on an internal psychological continuum. Because of internal noise factors' acting on the transmission of number information, the internal representation of number is described by a random variable:

$$
\mathrm{n} \mid \rightarrow \frac{1}{\sigma_{\mathrm{n}} \sqrt{2 \pi}} \exp -\frac{\left(\mathrm{q}_{\mathrm{n}}-\mathrm{x}\right)^{2}}{2 \sigma^{2}}
$$

Now, supposing that the subject is briefly presented a display containing $\mathbf{n}$ dots, we can specify the probability that the subject will report the correct number $\mathrm{n}$ as follows:

$$
P_{I}(n \mid n)=\frac{1}{\sigma_{n} \sqrt{2 \pi}} \int_{C(n ; n-1)}^{C(n ; n+1)} \exp -\frac{\left(q_{n}-x\right)^{2}}{2 \sigma_{n}^{2}} d x .
$$

The subscript $I$ in the conditional probability $P_{I}(n \mid n)$ indicates that all integer values are alternatives. The lower and upper integral limens, $C(n ; n-1)$ and $C(n ; n+1)$, denote the category bounds for correct responses to $n$.

It is not important to know, a priori, the precise form of the psychological function $q_{n}$ in Equation 2. Any positive monotonic function could be reasonable. Here, it is assumed that $q_{n}$ is a logarithmic function. This choice satisfies Fechner's law as it was incorporated in Thurstone's (1929) theory of comparative judgments of visual numerosity. It then follows that the category bounds $C(n ; n-1)$ and $\mathrm{C}(\mathrm{n} ; \mathrm{n}+1)$ are positioned exactly halfway between the internal representations of $n$ and $n-1$ for $C(n ; n-1)$ and $n$ and $n+1$ for $C(n ; n+1)$ (Parducci, 1963). Consequently, Equation 2 can be rewritten as:

$$
\begin{aligned}
& P_{I}(n \mid n) \\
& =\frac{1}{\sigma_{n} \sqrt{2 \pi}} \int_{1 / 2[\ln (n)+\ln (n-1)]}^{1 / 2[\ln (n)+\ln (n+1)]} \exp -\frac{[\ln (n)-x]^{2}}{2 \sigma_{n}^{2}} d x .(3)
\end{aligned}
$$

By substituting $y$ for

$$
\frac{\ln (n)-x}{\sigma_{n}}
$$

it follows directly that:

$$
P_{I}(n \mid n)=\frac{1}{\sqrt{2 \pi}} \int_{\frac{1}{2 o_{n}} \ln \left(\frac{n-1}{n}\right)}^{\frac{1}{2 o_{n}} \ln \left(\frac{n+1}{n}\right)} \exp \left(-\frac{y^{2}}{2}\right) d y .
$$

Note that

$$
\frac{1}{2 \sigma_{n}} \ln \left(\frac{n+1}{n}\right) \text { and } \frac{1}{2 o_{n}} \ln \left(\frac{n-1}{n}\right)
$$

really are $\mathrm{z}$ scores, because they are the integral limits of a standardized normal distribution. For reasons of parsimony, we assume the dispersion value $\sigma_{n}$ to be constant for all $\mathrm{n}, \sigma_{\mathrm{n}} \equiv \sigma$, that is, $\sigma_{\mathrm{n}}$ is only affected by internal noise due to momentary fluctuations of the organism regardless of the magnitude of $n$. To get some idea of what value the dispersion $\sigma$ of the internal representation should have, we can estimate $\sigma$ from the frequently reported finding that $P_{I}(7 \mid 7)=$ .5 (see, for instance, Averbach, 1963; Hunter \& Sigler, 1940). From

$$
P_{I}(7 \mid 7)=\frac{1}{\sqrt{2 \pi}} \int_{\frac{1}{20} \ln \left(\frac{6}{7}\right)}^{\frac{1}{20} \ln \left(\frac{8}{7}\right)} \exp \left(-\frac{y^{2}}{2}\right) d y=.5,
$$

we then find $o=.1080$. With this value for $\sigma$, we can determine $P_{I}(n \mid n)$ for all $n$. Figure 1 shows these conditional probabilities as a function of $n$.

In a situation where $\mathrm{n}$ is briefly presented, and the choice is between two alternatives, $n$ and $m$, the probability that a subject will respond with $n$ is:

$P_{n, m}(n \mid n)=-\frac{1}{\sqrt{2 \pi}}-\int_{-\infty}^{\frac{1}{20} \ln \left(\frac{m}{n}\right)} \exp \left(-\frac{y^{2}}{2}\right) d y$,

for $\mathrm{m}>\mathrm{n}$

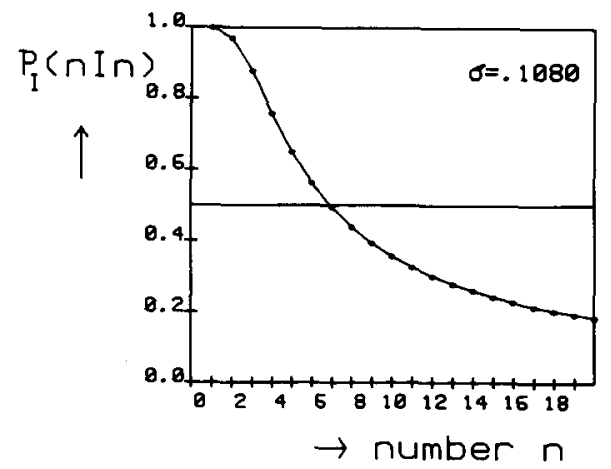

Figure 1. $P_{r}(n \mid n)$ plotted as a function of $n$, for $a=.1080$. 
and

$$
\begin{aligned}
P_{n, m}(n \mid n)= & \frac{1}{\sqrt{2 \pi}} \int_{\frac{1}{2 \sigma} \ln \left(\frac{m}{n}\right)}^{\infty} \exp \left(-\frac{y^{2}}{2}\right) d y, \\
& \text { for } m<n
\end{aligned}
$$

Defining "max" for the maximum value of $n$ and $m$, and "min" for the minimum value of both, we can then write the general expression for the conditional probability that a response $n$ will be given when $n$ is presented in the presence of an alternative $\mathrm{m}$ :

$$
P_{n, m}(n \mid n)=\frac{1}{\sqrt{2 \pi}} \int_{\frac{1}{2 \sigma} \ln \frac{\min }{\max }}^{\infty} \exp \left(-\frac{y^{2}}{2}\right) d y .
$$

$P_{n, m}(n \mid n)$ is illustrated in Figure 2 for $n=12$, alternative $m=10$, and $\sigma=.1080 . P_{12,10}(12 \mid 12)$ is the surface under the normal distribution function

$$
f(12)=\frac{1}{\sigma \sqrt{2 \pi}} \exp -\frac{[\ln (12)-x]^{2}}{2 \sigma^{2}}
$$

extending from $1 / 2 \ln (10 \cdot 12)$ to infinity. The Weber threshold is defined usually as the $50 \%$ correct discrimination between two stimuli. Operationally, this difference limen is a stimulus difference that is noticed $75 \%$ of the time. From $P_{n, m}(n \mid n)=.75$, we can derive $(1 / 2 \sigma) \ln (\max / \mathrm{min})$, from which the Weber fraction for visual number, $W_{b}=(\max -\min ) / \min$ is easily calculated. It should be noted that the limen to the left (smaller numbers) of an actually presented number is closer to that number than the limen to the right of it (larger numbers). More specifically, defining $n_{1}$ as that number, smaller than $n$, that can be discriminated from $n 50 \%$ of the time, we get for the Weber fraction:

$$
\mathrm{Wb}=\frac{\mathrm{n}-\mathrm{n}_{1}}{\mathrm{n}_{1}}
$$

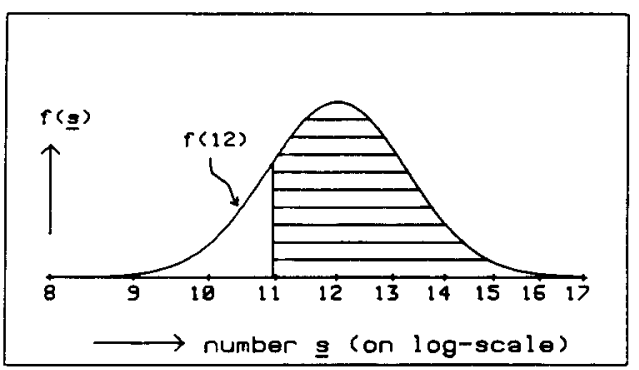

Figure 2. $P_{12,10}(12 \mid 12)$ is the surface under the normal distribution function $(1 / \sigma \sqrt{2 \pi}) \exp -\left\{[\ln (12)-x]^{2}\right\} / 2 \sigma^{2}$ extending from $1 / 2 \ln (10 \cdot 12)$ to infinity. Again, $\sigma=.1080$.
Defining $n_{r}$ as that number, larger than $n$, that can be discriminated from $n 50 \%$ of the time, we get:

$$
\mathrm{Wb}=\frac{\mathrm{n}_{\mathrm{r}}-\mathbf{n}}{\mathrm{n}} .
$$

The Weber fraction is a constant, so that the relation

$$
\frac{\mathrm{n}-\mathrm{n}_{1}}{\mathrm{n}_{1}}=\frac{\mathrm{n}_{\mathrm{r}}-\mathrm{n}}{\mathrm{n}}
$$

must hold. While $n_{1}$ is smaller than $n$, it then easily follows that the limen to the left of $n, n-n_{l}$, is smaller than that to the right of $n\left(n_{r}-n\right)$. Moreover, a closer examination of the equation stated above leads to the following peculiar relation:

$$
n_{1} \cdot n_{r}=n^{2}
$$

or

$$
\frac{\mathrm{n}_{1}}{\mathrm{n}} \cdot \frac{\mathrm{n}_{\mathrm{r}}}{\mathrm{n}}=1
$$

This relation represents well the asymmetric character of number discrimination. For instance, if 25 is the number limen to the left of 30,36 should be the limen to the right of 30 because $25 \cdot 36=(30)^{2}$.

At this point, it is necessary to refer to an early study of Crossman (1956), in which the need for a quantitative measure of "discriminability" was pointed out. He considered two to be discriminated signals $S_{1}$ and $S_{2}$ as points in a space of one dimension located at the distances $x_{1}, x_{2}$ from the origin. The ease of distinguishing between $S_{1}$ and $S_{2}$ was expected to depend on the "distance" between $x_{1}$ and $\mathrm{x}_{2}$. In the case of numbers, he did know from experiments that the distance depended on the ratio rather than on the absolute differences between the numbers. So, he took logarithms and measure in the space of $\log x$. The "distance" then became

$$
D\left(S_{1}, S_{2}\right)=\left|\log x_{1}-\log x_{2}\right|=\left|\log \frac{x_{1}}{x_{2}}\right|
$$

This " $D$ function" gives the ease of discrimination between $S_{1}$ and $S_{2}$. The reciprocal of $D$ has been named a "confusion function," since it measures the tendency to confuse $S_{1}$ and $S_{2}$. From our model, it follows that the detectability $\mathrm{d}^{\prime}$ is $(1 / 2 \sigma)$ $\log (\max / \mathrm{min})$, which closely resembles the distance function $\mathrm{D}$ as formulated by Crossman.

The remainder of this article is a report of two experiments, one threshold experiment (Experiment 1) and one RT experiment (Experiment 2), in which the discrimination hypothesis was tested. 
Table 1

Within Each Row Are Schematized Test Numbers t That Are Neighboring Numbers of a Row-Specific $s$

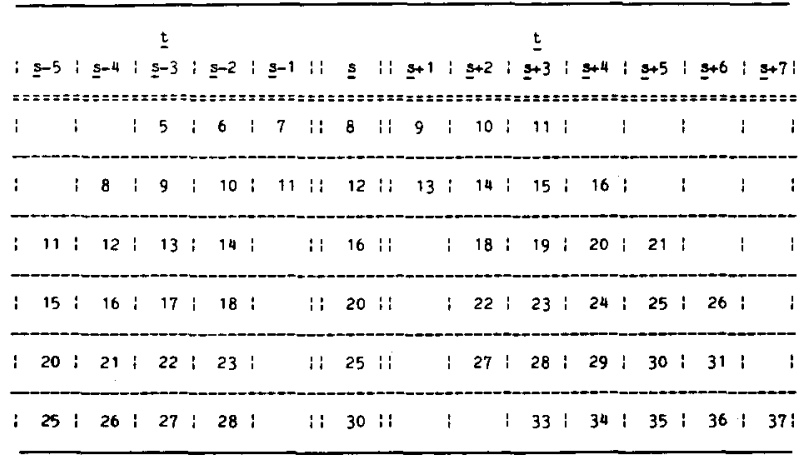

\section{EXPERIMENT 1}

\section{Method}

Subjects. Four (three males, 1 female) experimentally naive undergraduate psychology students were paid for their participation in the experiment.

Stimull. Dot patterns that differed in numbers of dots, as schematized in Table 1 , were constructed. Six numbers $(8,12,16$, 20,25 , and 30 ) are called standard numbrs, s; the other numbers are called test numbers, $t$. Within each row of Table 1 is presented one standard s, to the left and right of which are test numbers that are neighboring numbers of a row-specific $s$. For each number $\mathrm{n}, 30$ different configurations were constructed according to a pseudorandom procedure. This procedure started with partitioning a $10 \times 10$ square matrix into four quadrants. The $n$ dots of a particular stimulus were then placed randomly in the matrix cells with the constraint that each quadrant contained about the same number of dots. Each stimulus was unique with respect to its configuration. The stimuli are illustrated with three examples shown in Figure 3.

Procedure. Participants were tested individually in a quiet laboratory room. The stimuli were presented on a $27 \times 20 \mathrm{~cm}$ video monitor situated approximately $75 \mathrm{~cm}$ from the participant and at eye level. Dot diameter was $2 \mathrm{~mm}$, and the shortest of the distances from one dot to another was $8 \mathrm{~mm}$. Each time a sequence of $\mathbf{6 0}$ stimuli, consisting of 30 stimuli with number $t$ and 30 stimuli with $a$ to this $t$ belonging row specific number $s$ (see Table 1) was presented. Following the two-alternative forced-choice method, participants were told in advance what values $t$ and $s$ had, and were instructed to answer "yes" when s was presented and "no" when $t$ was presented. Order of presentation of the 60 stimuli was randomized. Each stimulus was presented for a fixed duration of $100 \mathrm{msec}$. The task was self-paced; a stimulus appeared on the screen 1,000 msec after the participant had pushed a button. The experimenter recorded the participants' responses. Whenever a participant committed an error, he or she received immediate verbal feedback ("wrong") from the experimenter. Following the 60 trials, the participant rested a few minutes. The next stimulus sequence contained 60 stimuli with a new $t$ and a new s. Each participant completed three sessions spread out over 2 or 3 consecutive days, with each session taking about $90 \mathrm{~min}$. The experiment was controlled by a PDP-11/45 system.

\section{Results}

The percentage of correct responses on any s, presented with each particular alternative $t$, was calculated over all subjects. The conditional chances, $\mathbf{P}_{\mathbf{s}, t}$ (s | s) were derived by dividing the percentages by 100. Figure 4 shows these frequencies as a function of $(t-s)$. In Figure 4, one can see that the profiles, each belonging to a particular $s$, expanded with increasing magnitude of $s$.

As is usually done, we chose the difference limen at the .75 level, which means that a difference between $s$ and $t$ is noticed $50 \%$ of the time. From Figure 4, limens were then measured at the intersection points of the profiles at the .75 chance level. In Figure 5, limens to the left (smaller numbers) and to the right of $s$ (larger numbers) are plotted as a function of $s$. One can see that for each $s$ the difference limen to the left was always smaller than the limen to the right of s. In Figure 5 the regression lines are also drawn. [Limens for larger numbers $(\mathrm{I})$, rho $=.96$; limens
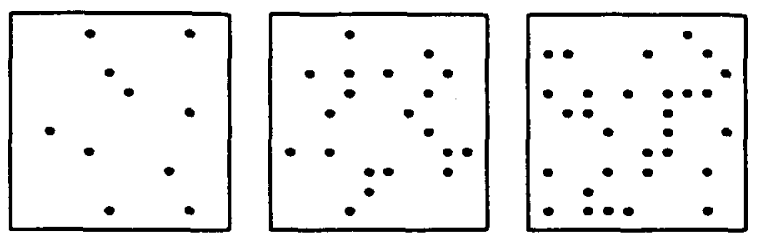

Figure 3. Three examples of stimuli used in the experiments.

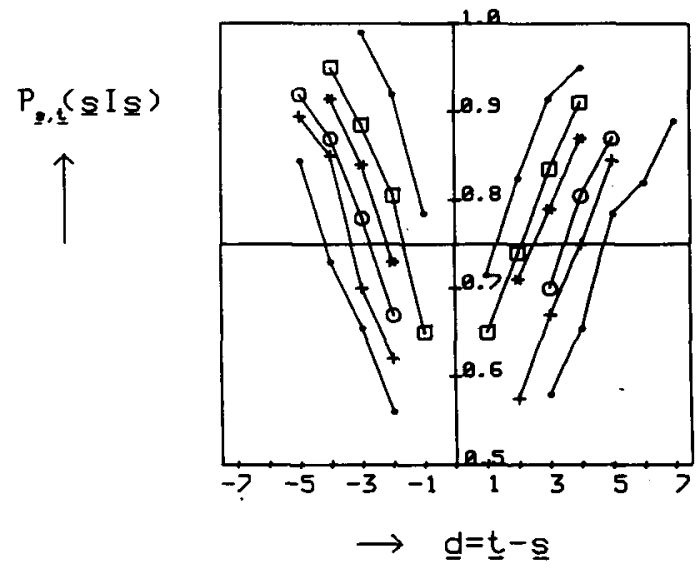

Figure 4. $P_{s, t}(s \mid s)$ as a function of $d=t-s . s=8(\cdot), s=12(\square)$, $s=16(*), s=20(O), s=25(+), s=30(\cdot)$.

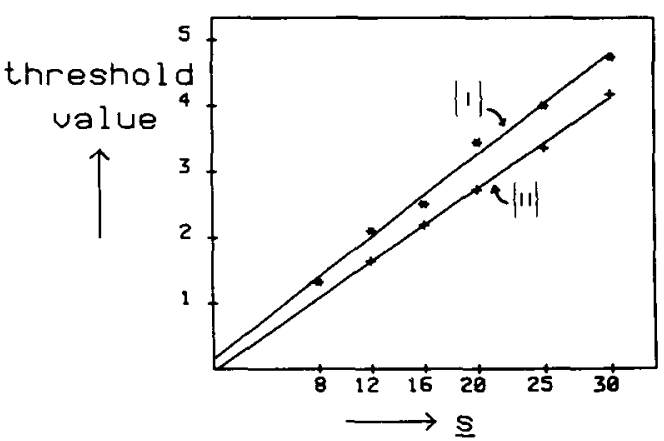

Figure 5. Threshold values to the left $(*)$ and to the right $(+)$ are plotted as a function of $s$. The corresponding regression lines are also drawn. 
for smaller numbers (II), rho $=.95]$. From regression line II, we derived $(\max -\min ) / \min =.164$. From regression line $I$, we derived $(\max -\min ) / \max =.139$, from which we calculated that $(\max -\min ) / \min =$ .162. We obtained the Weber fraction $\mathrm{dS} / \mathrm{S}=(\max -$ $\mathrm{min}) / \mathrm{min}$ as the mean of both values resulting from I and II: $W_{b}=.163$. From the relative limens to the right, .164, and to the left, .139, of a particular number, we obtained $\left(n_{1} / n\right)\left(n_{r} / n\right)=1.164 \times .861=1.0022$.

In Figure $6 P_{s, t}(s \mid s)$ is plotted as a function of $\max / \min$. In Equation 8, we postulated a relation between $\mathrm{P}_{\mathrm{s}, \mathrm{t}}(\mathrm{s} \mid \mathrm{s})$ and $\mathrm{max} / \mathrm{min}$. A likelihood procedure was used here to determine the value of the dispersion $\sigma$, given the chances $\mathrm{p}_{\mathrm{s}, \mathrm{t}}(\mathrm{s} \mid \mathrm{s})$ and the ratio $\max / \min$. A value of .1317 was found for $o$. With this value, the chances $P_{s, t}(s \mid s)$ were recalculated as a function of $\max / \mathrm{min}$ and plotted (solid curve) in Figure 6 as the best fit through the measured $P_{s, t}$ (s $\mid \mathrm{s})$. We then determined the value $\mathrm{max} / \mathrm{min}$ for which $P_{s, t}(s \mid s)=.75$. This ratio was found to be 1.162 , from which the Weber fraction $\mathrm{dS} / \mathrm{S}=(\max -$ $\mathrm{min}) / \mathrm{min}=.162$ was easily calculated.

To examine the hypothesis that a normal distribution function should underlie the discrimination process, we plotted the chances $P_{s, t}(s \mid s)$ on a probabilistic scale as a function of $(1 / 2 \sigma) \ln (\max / \mathrm{min})$ (see Figure 7). This is identical to transforming $P_{s, t}(s \mid s)$ to $\mathrm{z}$ scores and then plotting them on a linear scale. It should be noted that $(1 / 2 \sigma) \ln (\mathrm{max} / \mathrm{min})$ are also $z$ scores. As we can see, there exists a strong linear relation between the transformed $\mathrm{P}_{\mathrm{s}, \mathrm{t}}(\mathrm{s} \mid \mathrm{s})$ and $(1 / 2 \sigma) \ln (\mathrm{max} / \mathrm{min})$, a finding that confirms the normal distribution hypothesis.

The analysis of the results presented above was based upon the assumption that the psychological transform from external stimulus value $n$ to internal representation $\mathrm{q}_{\mathrm{n}}$ was logarithmic in nature (i.e., Fechner's law was satisfied). If a less stringent assumption had been made, fulfilling only the property of positive monotonicity, then conjoint measurement methods could be used to specify the nature of the function in question and to test equality of variances. If it is assumed that the cumulative distribution function can be approximated by the logistic function $F(x)=\left(1+e^{-x}\right)^{-1}$, then analysis of variance methods can be used to test the additivity properties. Both of these tests would require a factorial design.

The differential sensitivity of visual number can also be studied by letting each stimulus be present on the monitor until the subject gives the response. In this case, any possible strategy such as counting, subitizing, or estimating is applicable. It is reasonable to suppose that the selection of the strategy will be determined largely by whether the difference between two numbers to be discriminated is above threshold. Consider two stimuli comprising 20 and 30 dots. From Experiment 1, we know that they are easy to discriminate from each other. Therefore, one should

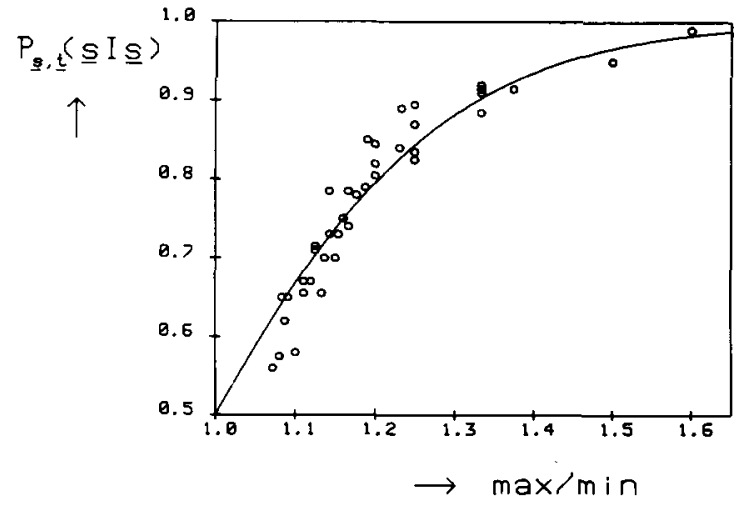

Figure 6. $P_{s, 1}(s \mid s)$ plotted as a function of $\mathrm{max} / \mathrm{min}$.

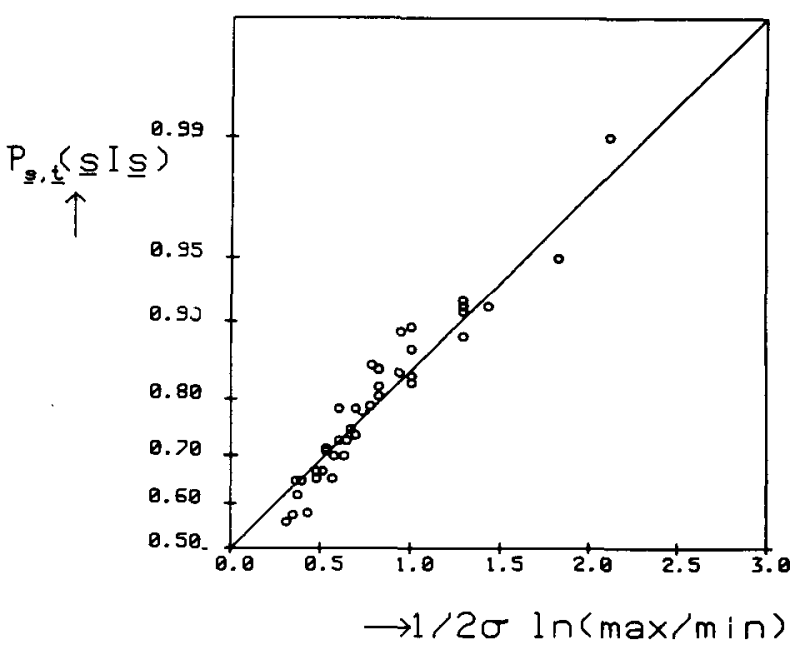

Figure 7. $P_{s, t}(s \mid s)$ plotted on a probability scale as a function of $(1 / 2 o) \ln (\max / \mathbf{m i n})$.

expect very short latencies as well as very high accuracy. On the other hand, if 29 dots are presented for discrimination from 30 , then only the strategy of counting would produce the correct response, a strategy that would increase response time drastically. Thus, somewhere between 20 and 29 , there is one number (or more) which, in direct comparison with $n=30$, will sometimes be estimated and sometimes be counted. Consequently, one might expect that latencies are distributed according to a bimodal distribution, one peak representing the counting times and the other representing immediate estimation times.

\section{EXPERIMENT 2}

\section{Method}

Subjects. Two young adults took part in the experiment and were paid for their participation.

Stimuli. The stimuli were the same as those use in Experiment 1 , with the exception that the standards, $s$, took the values of 8,20 , and 30 . Consequently, only neighboring numbers of these standards served as test stimuli, $t$ (see Table 1 ). 
Procedure. The procedure was in all respects identical to the one followed in Experiment 1, except for the following: Each stimulus now remained visible until the response, mediated by a microphone (Sennheiser headset), had surpassed a previously selected critical level. The subject was instructed to respond with "yes" whenever $s$ was presented and with "no" whenever $t$ was presented. He or she was asked to respond as accurately and as rapidly as possible. Latencies were registered automatically. The experimenter, who had a list of stimulus specifications, scored each response according to whether it was correct or incorrect, and then entered it into the computer. Whenever the participant committed an error, he or she received immediate feedback ("wrong") from the experimenter. Prior to each session, a sequence of stimuli containing only one dot was given. In order to determine the eventual latency differences with respect to verbalization of the response in question, participants responded yes or no in an alternate manner to these single dot stimuli.

\section{Results}

The analysis was based on all correct responses given to presented s stimuli. The frequency of incorrect responses was in general not more than $10 \%$ and typically occurred to stimuli near threshold. Figure 8 shows, for $s$ values of 8,20 , and 30 , the mean reaction times as a function of the difference $t-s$. As we can see in Figure 8, there was not only a decrease in RT as a function of increase in d, but also marked differences in the absolute magnitude of the latencies. In Figure 9, the standard deviations (SD) of the means depicted in Figure 8 are plotted as a function of $d$. One can see that, for $s=20$ and $s=30$, deviations culminated at values of $d$ which were roughly the same as values for the limens derived in Experiment 1. Finally, the distribution of reaction times is illustrated for $s$ as compared with three different test stimuli $(t=23,25$, and 27$)$, which were, respectively, above, around, and below discrimination threshold (Figure 10). While the distribution for the conditions of $s, t=(30,23)$ and $s, t=(30,27)$ point to a unimodal pattern, it is apparently dichotomized in the case of $s, t=(30,25)$.

\section{DISCUSSION}

The results of Experiment 2 agree with the findings of Experiment 1, both with respect to the predicted threshold location and the asymmetry of the rightand left-handed thresholds. Threshold values measured in Experiment 2 were, as a rule, slightly smaller than those obtained in Experiment 1, which probably reflects procedural differences between the two experiments. In the situation of Experiment 2, in which the participant had sufficient time to process the visual information and had also been instructed to give the correct answer as rapidly as possible, he or she would probably choose a safe strategy (counting) rather than a risky one (estimating). The bimodal shape of the distribution of latencies in those situations in which the difference between the numbers was around threshold suggested that the subject fol-

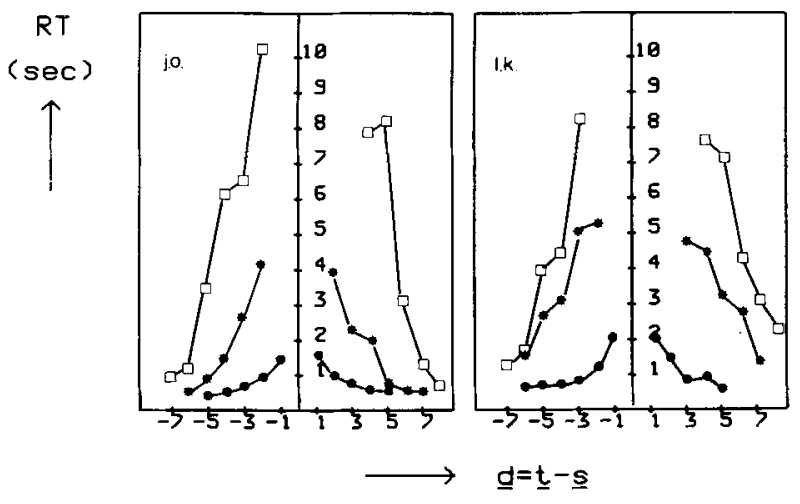

Figure 8. For two subjects, mean reaction time plotted as a function of $d=t-s . S=8(\cdot), s=20(*), s=30(\square)$.

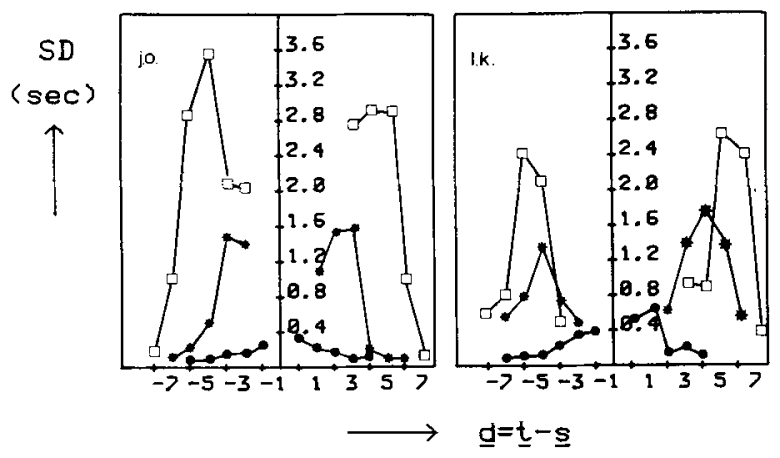

Figure 9. Standard deviations of the means depicted in Figure 8, plotted as a function of $d=t-s$.

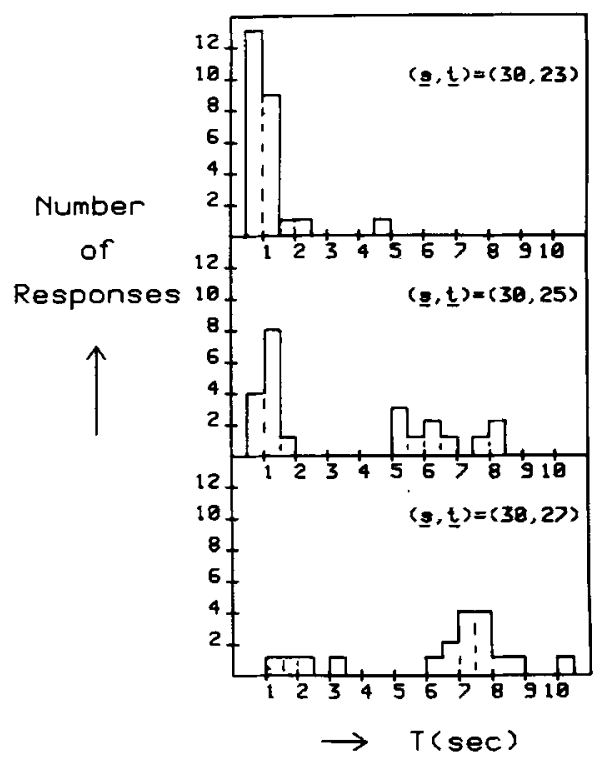

Figure 10. Dhtribution of reaction times on $s=30$ for the condltons $s, t=(30,23),(30,25)$, and $(30,27)$. 
lowed a strategy of counting roughly half of the time and a strategy of estimation half of the time. It is also possible, however, that the bimodal shape was affected by shifts in speed-accuracy criteria. In order to control for this rather awkward phenomenon, a much more laborious experimental and analytical design would be needed. Thus, one could vary systematically speed-accuracy instructions for the same task and simultaneously fit frequencies of correct responses and $R T$ data.

The results of Experiments 1 and 2 support Averbach's hypothesis that the span of visual apprehension is limited by the mutual discriminability of visual numbers. Pairwise discrimination between the small numbers $1,2,3,4,5$, and 6 can be done easily, because their ratio $(\max -\mathrm{min}) / \mathrm{min}$ lies well above the Weber fraction of .162. The numbers 6 and 7 can also be discriminated from each other more than $50 \%$ of the time, but not 7 and 8 . There is confusion between 7 and 8 more than $50 \%$ of the time. This result led us to conclude that the number six should be the upper limit of the span of apprehension. However, in an imaginary situation in which only even numbers are possible, the numbers $2,4,6,8,10$, and 12 can all be distinguished from each other over $50 \%$ of the time. In that case, the number 12 should be the upper limit of visual apprehension. Moreover, any pairwise discrimination between two numbers of which the ratio $(\max -\min ) / \min$ lies above the Weber fraction can be made more than $50 \%$ of the time. Therefore, our results strongly support Crossman's (1956) conclusion that the idea that the mind can grasp only a small number of objects at once remains quite unsupported by the evidence, if indeed it has any meaning at all.

So far, we have neglected the influence of pattern on number discrimination. Sometimes a $100 \%$ correct identification of four dots has been found (Kaufman et al., 1949), while we found $P_{I}(4 \mid 4)=$ .76, a value deduced from discrimination results of the larger numbers 16 and 20 , or 20 and 25 . But, for these larger numbers, pattern recognition does not make much sense. Pattern recognition becomes relevant, however, in the case of very small numbers: three dots nearly always make a triangle, for example, and four may often make a recognizable quadrilateral (Neisser, 1966, p. 42). Thus, apart from pure-number discrimination based on probability concepts, discrimination between small numbers can be facilitated by pattern effects.

Larger numbers of dots $(n>10)$ have been shown to be underestimated (Indow \& Ida, 1977). In pairwise comparison between two numbers, the discrimination is hardly influenced by underestimation because both numbers are underestimated. However, direct identification should lead to more erroneous results when $n>10$, so $P_{I}(n \mid n)$ should then be smaller, as depicted in Figure 1. To avoid these un- derestimation problems, a correction to our discrimination model can be made by substituting for the objective number $n$ (numerosity) its subjective equivalent $m$ (numerousness). For instance, Indow and Ida showed that, for randomly arranged dot patterns with $n>10$, the subjective number $m$ was exponentially related to the numerosity $n$ as $m=n^{.87}$.

Both factors, pattern recognition when $n \leqslant 4$ and underestimation when $\mathrm{n}>10$, do not make much sense when the number of dots is six, so we may safely retain the conclusion that the upper limit of the span of apprehension $(n=6)$ is due to discrimination on a probability basis only.

It is interesting to discuss our discrimination model in relation to the findings of Hunter and Sigler (1940). They showed that it took more light to see two black dots than it did to see one dot. Moreover, to reach a $50 \%$ level of correct responses on plates containing two or more dots, successively greater amounts of light were needed. The Bunson-Roscoe law, $I \cdot t=$ constant, was valid here for numbers of dots up to $n=8$. In our experiments, intensity was far above threshold. The only source of noise we assumed was in the transmission of number information appearing as a constant dispersion, $\sigma$, in the internal representation of number. But, if we should decrease intensity, it might well be possible that external noise relatively increases which emerges as an extra noise component, $\sigma_{\mathrm{e}}$, in our internal representation, so $o \mid \rightarrow \sigma_{i}+\sigma_{\mathrm{e}}$. $\mathbf{P}_{\mathrm{I}}(\mathrm{n} \mid \mathrm{n})$ then decreases monotonically with increasing $\sigma$ and, therefore, becomes a function of both intensity $I$ and exposure time $t$. It would be interesting to investigate the proper relationship between the dispersion $\sigma$ and intensity and/or exposure time.

At this point, we would like to say a word about the end effects that are frequently reported in number-naming tasks. For instance, Averbach (1963) used a limited set of response alternatives, the numbers 1 to 13 , and the task was to ascertain the briefly presented numbers of dots. To him, it seemed difficult to explain the constant superiority of 13 over 12 and of 12 over 11 , not only in terms of more correct, but in having fewer false alarms as well. In our model, $P_{1}(n \mid n)$ is the frequency of correct responses to $n$, while $n$ could be any integer. Limiting the set of alternatives should improve the task for numbers at the end of this limited set. While $P_{I}(13 \mid 13)$ is represented by the surface of the standard normal distribution function, extending from $(1 / 2 \sigma) \ln (12 / 13)$ to $(1 / 2 \sigma) \ln (14 / 13), P_{n=1, \ldots, 13}(13 \mid 13)$ is represented by the surface, extending from $(1 / 2 \sigma) \ln (12 / 13)$ to infinity, so $P_{n=1, \ldots, 13}(13 \mid 13)>P_{I}(13 \mid 13)$. Or, saying it with words, the transformation to internal representation admits a chance of responding 14 when 13 are presented, but the subject knows from instruction (or else, from experience) that there is no 14 , so he responds with 13 . All possible responses 
larger than 13 become 13 . Of course, this superiority of responding with 13 might bias the response of 12 over 11.

Many studies have dealt with the immediate apprehension of number. It was assumed that there was some number, $n$, of discrete objects that the mind could immediately perceive. The empirical question, then, was the value of $n$. In general, little discussion was devoted to the actual phenomenon. For instance, Beckwith and Restle (1966) merely called the underlying process "a somewhat mysterious but very rapid and accurate perceptual method." Finally, in this study we have shown that "subitizing" (Kaufman et al., 1949), "the span of apprehension" (Averbach, 1963), "the span of attention" (Fernberger, 1921; Freeman, 1912), and "the span of discrimination" (Hunter \& Sigler, 1940) all point to the same hypothetical construct, and that they do not refer to different behavioral phenomena. Number discrimination, and therefore number identification, is governed by probability concepts operating in a log space. Those numbers for which it mutually holds that $[(\max -\min ) / \min ]>w_{b}$, with $\max$ and $\min$ the larger and smaller numbers in question, can all be discriminated from each other more than $50 \%$ of the time. This set of numbers collapses to the classical span of apprehension when the difference between two neighboring numbers is restricted to one. These are the small numbers with an upper limit of six, for six can be discriminated from seven above threshold, but seven cannot from eight.

\section{REFERENCES}

Averbach, E. The span of apprehension as a function of exposure duration. Journal of Verbal Learning and Verbal Behavior, 1963, 2, 60-64.
Beckwith, M., \& Restle, F. Process of enumeration. Psychological Review, 1966, 73, 437-444.

Bourdon, B. Sur le temps nécessaire pour nominer les nombres. Revue Philosophique de la France et de l'Etranger, 1908, 33, 426-431.

Crossman, E. R. F. W. The measurement of discriminability. Quarterly Journal of Experimental Psychology, 1956, 7, 176-195.

Fernegraer, S. W. A preliminary study of the range of visual apprehension. American Journal of Psychology, 1921, 32, 121-133.

Freeman, F. N. Grouped objects as concrete basis for the number idea. Elementary School Teacher, 1912, 12, 306-314.

Hunter, W. S., Staler, M. The span of visual discrimination as a function of time and intensity of stimulation. Journal of Experimental Psychology, 1940, 26, 160-179.

Indow, T., IdA, M. Scaling of dot numerosity. Perception \& Psychophysics, 1977, 22, 265-276.

Kaufman, E. L., LoRd, M. W., Reese, T. W., \& Volkman, J. The discrimination of visual number. American Journal of Psychology, 1949, 62, 498-525.

Moyer, R. S., \& Landauer, T. K. The time required for judgments of numerical inequality. Nature, 1967, 215, 1519-1520.

Ne1sser, U. Cognitive psychology. New York: Appleton-CenturyCrofts, 1966.

Parducci, A. Range-frequency compromise in judgment. Psychological Monographs, 1963, 77 (2, Whole No. 565).

TAves, E. H. Two mechanisms for the perception of visual numerousness. Archives of Psychology, 1941, No. 265.

Thurstone, L. L. A law of comparative judgment. Psychological Review, 1927, 34, 273-286.

Thunstone, L. L. Fechner's law and the method of equalappearing intervals. Journal of Experimental Psychology, 1929, 12, 214-224.

Torgerson, W. S. Theory and methods of scaling. New York: Wiley, 1958.

Woodworth, R. S., \& Schloseerg, H. Experimental psychology. New York: Holt, Rinehart \& Winston, 1954.

(Manuscript received November 16, 1981; revision accepted for publication April 14, 1982.) 